\title{
Measurement of three-dimensional changes in lip vermilion in adult female patients after orthodontic extraction: a preliminary study
}

\section{Linhui Shen}

Peking University School of Stomatology https://orcid.org/0000-0003-4584-1092

Tian-yi Xie

Peking University School of Stomatology

Ruo-ping Jiang

Peking University School of Stomatology

Gui Chen

Peking University School of Stomatology

Tian-min Xu

Peking University School of Stomatology

Bing Han ( $\square$ kqbinghan@bjmu.edu.cn )

\section{Research article}

Keywords: lip vermilion, three-dimensional scanner, soft tissue, extraction treatment

Posted Date: April 24th, 2020

DOI: https://doi.org/10.21203/rs.3.rs-18817/v1

License: (9) This work is licensed under a Creative Commons Attribution 4.0 International License. Read Full License 


\section{Abstract \\ Background}

The objective of this study was to evaluate three-dimensional (3D) morphological changes in the lip vermilion in adult females after orthodontic treatment for four-premolar extraction in patients with dentoalveolar protrusion using a structured light-based scanner.

\section{Methods}

Forty-two female subjects with protruding lips were recruited as the treatment group; these patients underwent extraction of the four first premolars (PM1) without mini-implants for anchorage control. A total of twenty female subjects were enrolled in the non-treatment group; these patients did not require orthodontic treatment. Six facial landmarks (Ls, LI, R.Cu, L.Cu, R.Ch, L.Ch), three linear measurements (vermilion height, cupid's bow width, mouth width), and three area measurements (upper, lower, and total vermilion area along the 3D surface of the lip vermilion) were measured using 3D facial scans. Superimposed color maps and spectra were constructed for visual analysis of morphological changes in the lip vermilion for qualitative evaluation. The spacial deviations of the three volumetric measurements (upper, lower, and total vermilion) were constructed for quantitative analysis.

\section{Results}

Vermilion height and cupid's bow width decreased significantly and no significant change in mouth width was observed after extraction. The lower vermilion and total vermilion surface areas decreased significantly after orthodontic treatment in the extraction group, but the upper vermilion remained unchanged. Significant retractions were observed in vermilion volumetric measurements in the extraction group.

\section{Conclusions}

This study established a method to objectively and quantitatively compare the lip vermilion. Extraction of the four first premolars in adult females produced significant retraction in the lip vermilion. Morphological variation in the vermilion was different between the upper vermilion and the lower vermilion.

\section{Background}

Facial esthetics are important in social environments; thus, orthodontic treatment now places more emphasis on facial soft tissues, particularly the lips [1]. Malocclusion and procumbency of the lips are common chief complaints in the Asian population. Orthodontic extraction treatment is an effective way to relieve lip protrusion [2-5] because it can achieve better sagittal and frontal facial profiles. 
In the past, a reduction in lip protrusion following extraction of the premolars in patients with dentoalveolar protrusion was evaluated using two-dimensional (2D) imaging. lared et al. reported that maxillary and mandibular lip distances were retracted $1.3 \pm 1.8 \mathrm{~mm}$ and $1.2 \pm 1.4 \mathrm{~mm}$, respectively, after extraction [5]. The information provided by 2D imaging was limited in describing the border of the lip vermilion and lip vermilion changes were not confined in the projected plane. Therefore, morphological changes in the lip vermilion should be studied using three-dimensional (3D) techniques. 3D facial imaging devices such as stereophotogrammetry, laser scanning, and structured light scanning, have facilitated faster, noninvasive, and accurate measurements [6]. 3D measurements of the lips, including linear, area, and volumetric measurements, can be easily obtained and assessed using emerging 3D analysis software [6].

In facial soft tissues, the lip vermilion is a prominent feature for its obvious soft-tissue contours compared with facial skin [7]. Several studies explored the general 3D facial structures of Asian adult female patients and highlighted significant morphological influences in lip vermilion in patients who were considered more attractive than those who were considered average $[7,8]$. Several studies have demonstrated significant differences in lip vermilion curvature and shape during orthodontic treatment using 3D facial scanning $[9,10]$. However, the specific relationship between lip vermilion morphological changes in orthodontic extraction has not been fully explained, which makes the prediction of posttreatment vermilion morphology difficult. Thus, it is important to gain a better understanding of morphological changes in the lip vermilion when assessing the need for orthodontic extraction. The objective of the present study was to evaluate 3D morphological changes in the lip vermilion in adult females undergoing four-premolar extraction using a structured light-based scanner.

\section{Methods}

\section{Subjects}

Forty-two consecutive female subjects were recruited as the treatment group during their initial visit to the Department of Orthodontics, Peking University, School and Hospital of Stomatology, Beijing, China. The treatment group were aged between 22.1 and 28.9 years (mean $=25.2 \pm 1.9$ years). In the treatment group, all patients requiring extraction of four first premolars (PM1) without mini-implants were treated using the same fixed appliances $(0.022 \times 0.028$-inch bracket slot, Roth prescription; Xinya, Hangzhou, China) for at least 12 months. Twenty female subjects as the non-treatment group were enrolled among undergraduate students from Peking University, School and Hospital of Stomatology, Beijing, China. The non-treatment group were aged between 22.1 and 28.8 years (mean $=25.5 \pm 2.1$ years). All subjects had mild crowding or spacing $(<4 \mathrm{~mm}$ ), and the skeletal patterns were Class I or Class II (A point, nasion, B point [ANB] values $>1^{\circ}$ ). Other inclusion criteria were as follows: body mass index in the range of 18 to $24 \mathrm{~kg} / \mathrm{m}^{2}$, absence of posterior crossbite, no obvious facial asymmetry, no previous facial esthetic surgeries, no scars around the face, and overall good health. 
3D facial scans (Fig. 1) were available for each subject and acquired using a structured light-scanning system (accuracy: \pm 0.05 mm; 3D CaMega; Boweihengxin Technology Inc., Beijing, China). Subjects were asked to relax their lips and peri-oral muscles in a natural head position during facial scans. All subjects had T1 and T2 facial scans. In the treatment group, T1 was the pre-treatment time point and T2 was the post-treatment time point. In the non-treatment group, the duration between T1 and T2 was at least 12 months.

\section{Reconstruction and analysis of morphological changes in the lip vermilion}

\section{Coordinate system construction}

The coordinate system (Figs. 2A and 2B) was constructed using the method described by Alqattan et al. [11] using the software Geomagic Qualify 12 (3D Systems, Rock Hill, South Carolina). The point located halfway between the inner canthi of the eyes was taken as the origin of the coordinate system, at which three planes coincided. The sagittal plane $(\mathrm{YZ})$ was determined as the symmetry plane of the original mirror face structure. The transverse plane $(X Z)$ was constructed using the cylinder that fitted all data points of the original mirror face structure. The coronal plane $(X Y)$ was perpendicular to the sagittal and transverse planes.

\section{Superimposition and visual analysis of morphological changes in the lip vermilion}

The two selected digital facial scans (T1 and T2) in the coordinate system were superimposed according to the "best-fit alignment" algorithm [12]; thus, a superimposed 3D image with a unified system was obtained for each subject (Fig. 2C). Superimposed color maps and spectrums were constructed for visual analysis (Figs. 2D and 2E).

\section{Quantitative analysis for landmark identification, linear, and area measurements}

Table 1 provides abbreviations and definitions of each lip vermilion measurement. Six facial landmarks (Fig. 3A and Table 1) were marked on the 3D image, including two landmarks in the middle line (Ls, LI) and two bilateral landmarks (R.Cu, L.Cu, R.Ch, L.Ch). Three straight-line distances (Fig. 3A and Table 1) as the linear measurements (vermilion height, cupid's bow width, mouth width) were measured from 3D images. The 3D surfaces were marginated manually according to their vermilion anatomical morphologies and 3D surfaces (Figs. 3B-3D and Table 1) and area measurements (upper, lower, and total vermilion area along the 3D surface) were measured from 3D images.

\section{Set-up of the measuring planes and quantitative analysis of volumetric measurements}


The measuring plane was defined (Figs. 4A and 4B) for quantitative analysis of volumetric measurements. In the unified coordinate system of the superimposed 3D image, the measuring plane (Figs. 4C and 4D) was perpendicular to the $\mathrm{YZ}$ sagittal plane, which passed through the bilateral inner canthi point. The 3D surfaces were marginated manually according to vermilion anatomical morphology (Fig. 5A-5C), erasing the other non-vermilion part [13], and projected to the measuring planes, enabling three volumetric measurements (upper, lower, and total vermilion volumes projected to measuring planes) to be measured (Figs. 5D-5F). The space deviations as volumetric changes (T2 measurement - T1 measurement = space deviation) in the upper, lower, and total vermilion were constructed and used for quantitative analysis.

Table 1

Definition of lip vermilion measurements

Variable Definition

Landmarks in the midline

Ls

Labiale superius

LI

Most prominent point of the vermilion border of cupid's bow of the lower lip

Bilateral landmarks

R.Cu (right cupid's bow) Most prominent point of the vermilion border of right cupid's bow of the upper lip

L.Cu (left cupid's bow) Most prominent point of the vermilion border of left cupid's bow of the upper lip

R.Ch (right cheilion) Most lateral extent of the outline of the lip on the right side

L.Ch (left cheilion) Most lateral extent of the outline of the lip on the left side

Linear measurements

$(\mathrm{mm})$

Vermilion height

The straight-line distance between $\mathrm{LI}$ and Ls

Cupid's bow width

The straight-line distance between R.Cu and L.Cu

Mouth width

The straight-line distance between R.Ch and L.Ch

Area measurements

$\left(\mathrm{mm}^{2}\right)$

Upper vermilion area

Lower vermilion area

Total vermilion area
Area of the upper vermilion along the surface

Area of the lower vermilion along the surface

Area of the upper and lower vermilion along the surface 
Data were analyzed using SPSS software (version 23.0; IBM Corp., Armonk, NY, USA). All measurements were repeated by the same operator. The degree of intra-observer error was assessed by comparing the $x$, $y$, and $z$ components of each landmark (two midline and four lateral) with measurements on 20 subjects chosen randomly in over a two-week interval. The threshold for acceptable intra-observer error for each landmark was 0.90 with the calculation of intraclass correlation coefficient (ICC). The normality of the data from 3D facial scans was confirmed using the Shapiro-Wilk test. A t-test was performed to evaluate changes during orthodontic treatment. A p value of $<0.05$ was considered statistically significant.

\section{Results}

In the treatment group, the quantitative analysis of changes in landmark identification pre-treatment and post-treatment is showed in Table 2 (available in the Supplemental Files section). There were no significant changes $(p>0.05)$ in any of the assessments in the vertical dimension among all landmarks. There were significant changes in the sagittal dimension of points $\mathrm{LI}(p<0.01)$, R.Ch $(p<0.05)$, and L.Ch $(p<0.01)$. There were significant changes in the horizontal dimension of point R.Cu $(p<0.01)$ and L.Cu $(p$ $<0.05)$. Intra-observer reliability was estimated using the ICC for each landmark in three dimensions. In sum, the ICC values indicated excellent reliability (>0.90 for all calculations).

The quantitative analysis of changes in linear and area measurements pre-treatment versus posttreatment in the treatment group is shown in Table 3. Table 4 shows the comparison between the treatment group and the non-treatment group. For linear measurements, cupid's bow width and vermilion height decreased significantly during orthodontic treatment $(p<0.01$ and $p<0.05$, respectively) and mean differences of each from $\mathrm{T} 1$ to $\mathrm{T} 2$ were significantly different when comparing with the non-treatment group ( $p=0.01$ and $p<0.05$, respectively). No significant change was observed in mouth width. There were no significant differences in the area measurements of the upper vermilion, while lower vermilion area and total vermilion area were significantly decreased after orthodontic treatment $(p<0.01$ and $p<$ 0.05 , respectively) and mean differences of each were significantly different between the treatment and the non-treatment groups ( $p<0.05$ and $p<0.05$, respectively). 
Table 3

Comparison of changes in linear and area measurements pre- and post-treatment in the treatment group

\begin{tabular}{|c|c|c|c|}
\hline Variable & Pretreatment & Posttreatment & $p$-value \\
\hline \multicolumn{4}{|c|}{ Linear measurements (mm) } \\
\hline Cupid's bow width & $16.693 \pm 2.054$ & $15.756 \pm 1.888$ & $0.008^{\star \star}$ \\
\hline Mouth width & $50.506 \pm 4.144$ & $50.829 \pm 3.987$ & 0.488 (NS) \\
\hline Vermilion height & $21.887 \pm 2.941$ & $20.806 \pm 2.360$ & $0.027^{*}$ \\
\hline \multicolumn{4}{|c|}{ Area measurements $\left(\mathrm{mm}^{2}\right)$} \\
\hline Upper vermilion area & $683.633 \pm 156.501$ & $677.384 \pm 128.561$ & 0.752 (NS) \\
\hline Lower vermilion area & $638.434 \pm 125.582$ & $587.430 \pm 138.479$ & $0.003^{\star \star}$ \\
\hline Total vermilion area & $1200.085 \pm 196.620$ & $1130.273 \pm 243.082$ & $0.031^{*}$ \\
\hline
\end{tabular}

Table 4

Mean differences from T1 to T2 in linear and area measurements between treatment and non-treatment groups

\begin{tabular}{|llll|}
\hline Variable & Treatment group & Non-treatment group & p-value \\
\hline Linear measurements $(\mathrm{mm})$ & & & \\
\hline Cupid's bow width & $-0.937 \pm 2.168$ & $-0.010 \pm 0.365$ & $0.010^{*}$ \\
\hline Mouth width & $0.323 \pm 2.996$ & $0.009 \pm 0.587$ & $0.516(\mathrm{NS})$ \\
\hline Vermilion height & $-1.081 \pm 3.056$ & $0.103 \pm 0.319$ & $0.017^{\star}$ \\
\hline Area measurements $\left(\mathrm{mm}^{2}\right)$ & & & \\
\hline Upper vermilion area & $-6.250 \pm 127.373$ & $33.995 \pm 81.612$ & $0.139(\mathrm{NS})$ \\
\hline Lower vermilion area & $-51.005 \pm 104.876$ & $8.669 \pm 87.210$ & $0.023^{*}$ \\
\hline Total vermilion area & $-69.811 \pm 202.978$ & $52.115 \pm 156.475$ & $0.012^{*}$ \\
\hline $\begin{array}{l}\text { Values are presented as mean } \pm \text { standard deviation. An independent } \mathrm{t} \text {-test was used to compare the } \\
\text { treatment and non-treatment groups. NS, non-significant; }{ }^{*} \mathrm{p}<0.05,{ }^{*} \mathrm{p}<0.01 .\end{array}$ & \\
\hline
\end{tabular}

As shown in Fig. 6, superimposed color maps and spectrums were constructed for visual analysis of morphological changes in the lip vermilion during orthodontic therapy in the treatment group (Figs. 6A$6 \mathrm{H}$ ) and the observing duration in the non-treatment group (Figs. 6I-6P). Table 5 shows the comparison 
of volumetric changes between the treatment and non-treatment groups in the upper, lower, and total vermilion. There were significant differences in the three volumetric measurements $(p<0.05, p<0.01$, and $p<0.01$, respectively).

Table 5

Volumetric changes between the treatment and non-treatment groups

\begin{tabular}{|lccc|}
\hline Variable & Treatment group & Non-treatment group & p-value \\
\hline Upper vermilion volume & $-1625.808 \pm 2607.153$ & $280.513 \pm 2667.685$ & $0.012^{\star}$ \\
\hline Lower vermilion volume & $-1893.223 \pm 2196.202$ & $495.162 \pm 2568.108$ & $0.001^{\star *}$ \\
\hline Total vermilion volume & $-2933.143 \pm 5478.742$ & $1158.992 \pm 4654.831$ & $0.004^{\star *}$ \\
\hline $\begin{array}{l}\text { Values are presented as mean } \pm \text { standard deviation }\left(\mathrm{mm}^{3}\right) \text {. An independent t-test was used to } \\
\text { compare the treatment and non-treatment groups. NS, non-significant; }{ }^{\star} \mathrm{p}<0.05,{ }^{\star \star} \mathrm{p}<0.01 .\end{array}$ \\
\hline
\end{tabular}

\section{Discussion}

At present, most research methods used to measure the soft tissue morphology of the lips are based on 2D films and measurement indicators are not sufficiently comprehensive. Research into 3D measurements is scarce; most existing research into 3D measurements of 2D indicators does not fully reflect the morphological characteristics of the soft tissue of the lips and the changes that occur during orthodontic treatment. This is because facial soft tissue is an irregular curved surface. In many cases, soft tissue samples must reflect 3D features such as curved surfaces to enable a more accurate measure of morphology. In this study, 3D facial scanning technology was used to establish an appropriate measurement method, especially to measure changes in area and volume in the lip vermilion. The data collected using a 3D facial scanner was analyzed using Geomagic Qualify software; it was easy to quantify and describe morphological changes in the lip vermilion by establishing a coordinate system.

Adult female subjects who did not receive orthodontic treatment were enrolled in this study as the nontreatment group. In the present study, changes in cupid's bow width and vermilion height in the treatment group ( $p<0.01, p<0.05$, respectively) were consistent with those comparisons with the non-treatment group ( $p<0.05, p<0.05$, respectively). Changes in lower and total vermilion area in the treatment group ( $p$ $<0.01, p<0.05$, respectively) were also statistically consistent with the non-treatment group $(p<0.05, p<$ 0.05 , respectively). Masticatory muscle tension and physiological condition of lips were usually different at different times and might be possible influencing factors contributing to the accidental error of measurement and the variation in vermilion changes [10]. Those results in this study suggested that the changes of those measurements in the treatment group mainly due to the influence of extraction treatment, rather than being affected by the differences in time point for measurement.

In the present study, the statistical results of the linear and area measurements were considered in combination with the results of landmark identification. It was a preliminary study to the pattern of lip 
vermilion morphological change under extraction treatment. There was no significant change in mouth width, which may be related to the observation that R.Ch and L.Ch showed no significant changes in their horizontal dimension. The upper vermilion area was also not significantly difference, which may be associated with the fact that R.Cu, L.Cu, and Ls did not show significant changes in their sagittal dimension. Besides, changes in mouth width and upper vermilion area in the treatment group $(p=0.488$, $p=752$, respectively) were statistically consistent with those comparisons with the non-treatment group $(p=0.516, p=0.139$, respectively). Therefore, the accidental error of measurement could be considered to be negligible and mouth width and upper vermilion area appeared to be stable after the extraction treatment. A significant decrease in cupid's bow width may be related to the significant changes in R.Cu and L.Cu in the horizontal dimension. The lower vermilion area was significantly reduced, which may be associated with significant changes in R.Ch, L.Ch, and LI in the sagittal dimension. The significant decrease in vermilion height may be related to significant changes in $\mathrm{LI}$ in the vertical dimension. Trisnawaty et al. reported that vermilion height decreased by $0.39 \mathrm{~mm}$ as the linear measurement of StMe when the four first premolars were extracted [14]. However, Maltagliati et al. reported that lower lip height increased $(4.61 \pm 3.61 \mathrm{~mm})$ significantly and the vertical dimension of the upper lip was not modified in the treated group with extraction [15], which indicates that vermilion height was increased in their study. The controversy presented by those articles requires further investigation.

A qualitative impression was obtained using the superimposed color maps and spectra. As presented in Fig. 6, the vermilion area in the treatment group retracted backwards after orthodontic treatment and differed from the superimposed results observed in the non-treatment group. A statistically significant degree of retraction occurred in the vermilion $(p<0.01)$ after orthodontic extraction in the present study, which can be used as quantitative evidence to support the qualitative results of the superimposed color maps and spectra. Both the upper $(p<0.05)$ and lower $(p<0.01)$ vermilion were retracted in the treatment group. These results are in accordance with the clinical observations of historical studies $[4,16]$. Lee et al. reported that extraction of the four premolars led to significantly greater retraction of the upper/lower lip from Ricketts' E plane [17]. Liu et al. reported a significantly smaller lip vermilion after extraction, indicating that the lip vermilion was more slender when the vermilion height-width ratio was decreased [10]. Nevertheless, contradicting conclusions drawn by Freitas et al. [18] and Basciftci et al. [19] reported that extraction of first premolars did not imply a greater degree of retraction in lip soft tissue. A more harmonious vermilion was considered attractive and had positive effects on lip esthetics [5], but the relationship between lip morphology and facial esthetics is complex. Ethnicity, skin color, age, and profile characteristics might all affect the quality of treatment [20]. According to Kocadereli et al., for the sake of retraction in the upper and lower lips, extraction caused a harmful effect on facial esthetics, flattening the facial profile [21]. However, in cases of nose or chin protrusion, compensatory lip protrusion improves the profile attractiveness [22]. Compared with the area and volume measurement of upper vermilion retraction ( $-6.250 \mathrm{~mm}^{2}$ and $-1625.808 \mathrm{~mm}^{3}$, respectively) after extraction, the lower vermilion ($51.005 \mathrm{~mm}^{2}$ and $-1893.223 \mathrm{~mm}^{3}$, respectively) had a greater degree of retraction, suggesting that the lower vermilion plays a major role in retraction. These results are consistent with historical articles that studied the responding patterns of soft tissue. A recent systematic review reported that the average lip 
retraction after extraction of the 4 premolars was $1.4 \mathrm{~mm}$ for the upper vermilion and $2.0 \mathrm{~mm}$ for the lower vermilion [23]. The difference between the upper and lower vermilions may vary considerably with incisor variables [24], the distance of the mandibular lip to the esthetic line [5], and other factors.

One limitation of the present study was the small sample size and there was a remarkable variation of lip morphology between different populations [20]. Large values of standard deviations among people can be an evidence and the standard deviations of upper, lower and total vermilion, as the area measurement, were larger than $80 \mathrm{~mm}^{2}$ of all calculations in this study. Previous 3D studies found that vermilion height of Asian adult females was in the range of $16.0 \mathrm{~mm}$ to $19.0 \mathrm{~mm}[10,25,26]$ and the results in the present study were larger both in pre- and post-treatment $(21.887 \mathrm{~mm}, 20.806 \mathrm{~mm}$, respectively) in the treatment group. Although samples were enrolled in an effort to eliminate bias, heterogenous intervention could not be avoided, which is a common problem in clinical research.

Thus, this study was a preliminary exploration and the results should not be generalized to clinical orthodontic treatment as a whole (e.g., in the context of different malocclusions or different treatment methods). A larger sample size would be needed in further studies to clarify the findings. Possible influencing factors that could contribute to changes in the vermilion (e.g., facial soft tissue conditions and skeletal patterns) also require further investigation or verification.

\section{Conclusions}

The present study established a method to quantify and qualify possible changes in the lip vermilion during orthodontic extraction in female adult patients. Cupid's bow width, vermilion height and lower vermilion area appeared to decrease and mouth width and upper vermilion area appeared to be stable after the extraction treatment. Significant retraction of lip vermilion was observed and the lower vermilion area showed a greater degree of retraction compared with the upper vermilion. Further studies with a larger sample size and a multitude of parameters would help to verify the results of the present study.

\section{Abbreviations}

3D: three-dimensional; PM1: first premolar; Ls: Labiale superius; LI: Point of lower lip; R.Cu: Right cupid's bow; L.Cu: Left cupid's bow; R.Ch: Right cheilion; L.Ch: Left cheilion ICC: intraclass correlation coefficient; NS: non-significant;

\section{Declarations}

\section{Acknowledgments}

The authors thank Donghao Wei from the Department of Oral Implantology at Peking University School and Hospital of Stomatology in Beijing for his support with the techniques. 


\section{Author contributions}

Dr. Lin-hui Shen was a major contributor in conducting research and writing the manuscript. Dr. Lin-hui Shen collected data and analysed and interpreted the data. The authors thank Dr. Tian-yi Xie, Dr. Ruo-ping Jiang, and Dr. Gui Chen from the Department of Orthodontics at Peking University School and Hospital of Stomatology in Beijing for providing raw data and encouragement during the study. Dr. Tian-min Xu and Dr. Bing Han supervised the research and revised important parts of the manuscript. All authors read and approved the final manuscript.

\section{Funding}

This work was supported by grants from the National Natural Science Foundation of China (51672009, 51972005), Beijing Natural Science Foundation 7192227 and the Program for New Clinical Techniques and Therapies of Peking University School and Hospital of Stomatology (PKUSSNCT-19A05).

\section{Availability of data and materials}

The datasets used and/or analyzed during the current study are available from the corresponding author on reasonable request.

\section{Ethics approval and consent to participate}

This study was a retrospective study conducted in accordance with the declaration of Helsinki (1975, as revised in 2000). The study protocol was approved by the local ethics committee (Institutional Review Board of Peking University School and Hospital of Stomatology, approval number: PKUSSIRB201948110). All patients provided informed consent after a comprehensive consultation.

\section{Consent for publication}

Written informed consent to publish individual person's data (images) were obtained.

\section{Competing interests}

The authors declare that they have no competing interests. 


\section{References}

1. Fortes HNDR, Guimarães TC, Belo IML, Matta, E. N. R. D. Photometric analysis of esthetically pleasant and unpleasant facial profile. Dental Press J Orthod. 2014;19(2):66-75.

2. Zierhut EC, Joondeph DR, Artun J, Little RM. Long-term profile changes associated with successfully treated extraction and nonextraction Class II Division 1 malocclusions. Angle Orthod. 2000;70(3):208-19.

3. Ong HB, Woods MG. An occlusal and cephalometric analysis of maxillary first and second premolar extraction effects. Angle Orthod. 2001;71(2):90-102.

4. Moseling KP, Woods MG. Lip curve changes in females with premolar extraction or nonextraction treatment. Angle Orthod. 2004;74(1):51-62.

5. lared W, da Silva EMK, lared W, Macedo CR. Esthetic perception of changes in facial profile resulting from orthodontic treatment with extraction of premolars: a systematic review. J Am Dent Assoc. 2017;148(1):9-16.

6. Kau CH, Richmond S, Incrapera A, English J, Xia JJ. Three-dimensional surface acquisition systems for the study of facial morphology and their application to maxillofacial surgery. Int J Med Robot. 2007;3(2):97-110.

7. Tanikawa C, Nakamura K, Yagi M, Takada K. Lip vermilion profile patterns and corresponding dentoskeletal forms in female adults. Angle Orthod. 2009;79(5):849-58.

8. Kim SY, Bayome M, Park JH, Kook YA, Kang JH, Kim KH, Moon HB. Evaluation of the facial dimensions of young adult women with a preferred facial appearance. Korean $\mathrm{J}$ Orthod. 2015;45(5):253-60.

9. Ahn HW, Chang YJ, Kim KA, Joo SH, Park YG, Park KH. Measurement of three-dimensional perioral soft tissue changes in dentoalveolar protrusion patients after orthodontic treatment using a structured light scanner. Angle Orthod. 2014;84(5):795-802.

10. Liu ZY, Yu J, Dai FF, Jiang RP, Xu TM. Three-dimensional changes in lip vermilion morphology of adult female patients after extraction and non-extraction orthodontic treatment. Korean $\mathrm{J}$ Orthod. 2019;49(4):222-34.

11. Alqattan M, Djordjevic J, Zhurov Al, Richmond S. Comparison between landmark and surface-based three-dimensional analyses of facial asymmetry in adults. Eur J Orthod. 2015;37(1):1-12.

12. González-Martín O, Veltri M, Moraguez O, Belser UC. Quantitative three-dimensional methodology to assess volumetric and profilometric outcome of subepithelial connective tissue grafting at pontic sites: a prospective pilot study. Int J Periodontics Restorative Dent. 2014;34(5).

13. Tian J, Wei D, Zhao Y, Di P, Jiang X, Lin Y. Labial soft tissue contour dynamics following immediate implants and immediate provisionalization of single maxillary incisors: A 1-year prospective study. Clin Implant Dent Relat Res. 2019;21(3):492-502.

14. Trisnawaty N, loi H, Kitahara T, Suzuki A, Takahashi I. Effects of extraction of four premolars on vermilion height and lip area in patients with bimaxillary protrusion. Eur J Orthod. 2013;35(4):521-8. 
15. Maltagliati L, Henriques JFC, Janson G, Almeida RRD, Freitas MR. D. Influence of orthopedic treatment on hard and soft facial structures of individuals presenting with Class II, Division 1 malocclusion: a comparative study. J Appl Oral Sci. 2004;12(2):164-70.

16. Yasutomi H, loi H, Nakata S, Nakasima A, Counts AL. Effects of retraction of anterior teeth on horizontal and vertical lip positions in Japanese adults with the bimaxillary dentoalveolar protrusion. Orthod Waves. 2006;65(4):141-7.

17. Lee SH, Chung DH, Cha KS, Lee JW, Lee SM. The Esthetic Impact of Extraction and Nonextraction Treatments on Korean People. J Dent Rehabil Appl Sci. 2013;29(2):119-26.

18. Freitas BV, Rodrigues VP, Rodrigues MF, de Melo HV, dos Santos PC. Soft tissue facial profile changes after orthodontic treatment with or without tooth extractions in Class I malocclusion patients: A comparative study. J Oral Biol Craniofac Res. 2019;9(2):172-6.

19. Ayhan Basciftci F, Uysal T, Buyukerkmen A, Demir A. The influence of extraction treatment on Holdaway soft-tissue measurements. Angle Orthod. 2004;74(2):167-73.

20. López-Mateos MM, Carreño-Carreño J, Palma JC, Alarcón JA, López-Mateos CM, Menéndez-Núñez M. Three-dimensional photographic analysis of the face in European adults from southern Spain with normal occlusion: reference anthropometric measurements. BMC Oral Health. 2019;19(1):196.

21. Kocadereli I. Changes in soft tissue profile after orthodontic treatment with and without extractions. Am J Orthod Dentofac Orthop. 2002;122(1):67-72.

22. Torsello F, Graci M, Grande NM, Deli R. Relationships between facial features in the perception of profile attractiveness. Prog Orthod. 2010;11(2):92-7.

23. Konstantonis D, Vasileiou D, Papageorgiou SN, Eliades T. Soft tissue changes following extraction vs. nonextraction orthodontic fixed appliance treatment: a systematic review and meta-analysis. Eur J Oral Sci. 2018;126(3):167-79.

24. Kim HH, Lee JW, Cha KS, Chung DH, Lee SM. Three-dimensional assessment of upper lip positional changes according to simulated maxillary anterior tooth movements by white light scanning. Korean J Orthod. 2014;44(6):281-93.

25. Jayaratne YS, Deutsch CK, Zwahlen RA. A 3D anthropometric analysis of the orolabial region in Chinese young adults. Br J Oral Maxillofac Surg. 2013;51(8):908-12.

26. Wong WW, Davis DG, Camp MC, Gupta SC. Contribution of lip proportions to facial aesthetics in different ethnicities: a three-dimensional analysis. J Plast Reconstr Aesthet Surg. 2010;63(12):20329.

\section{Figures}




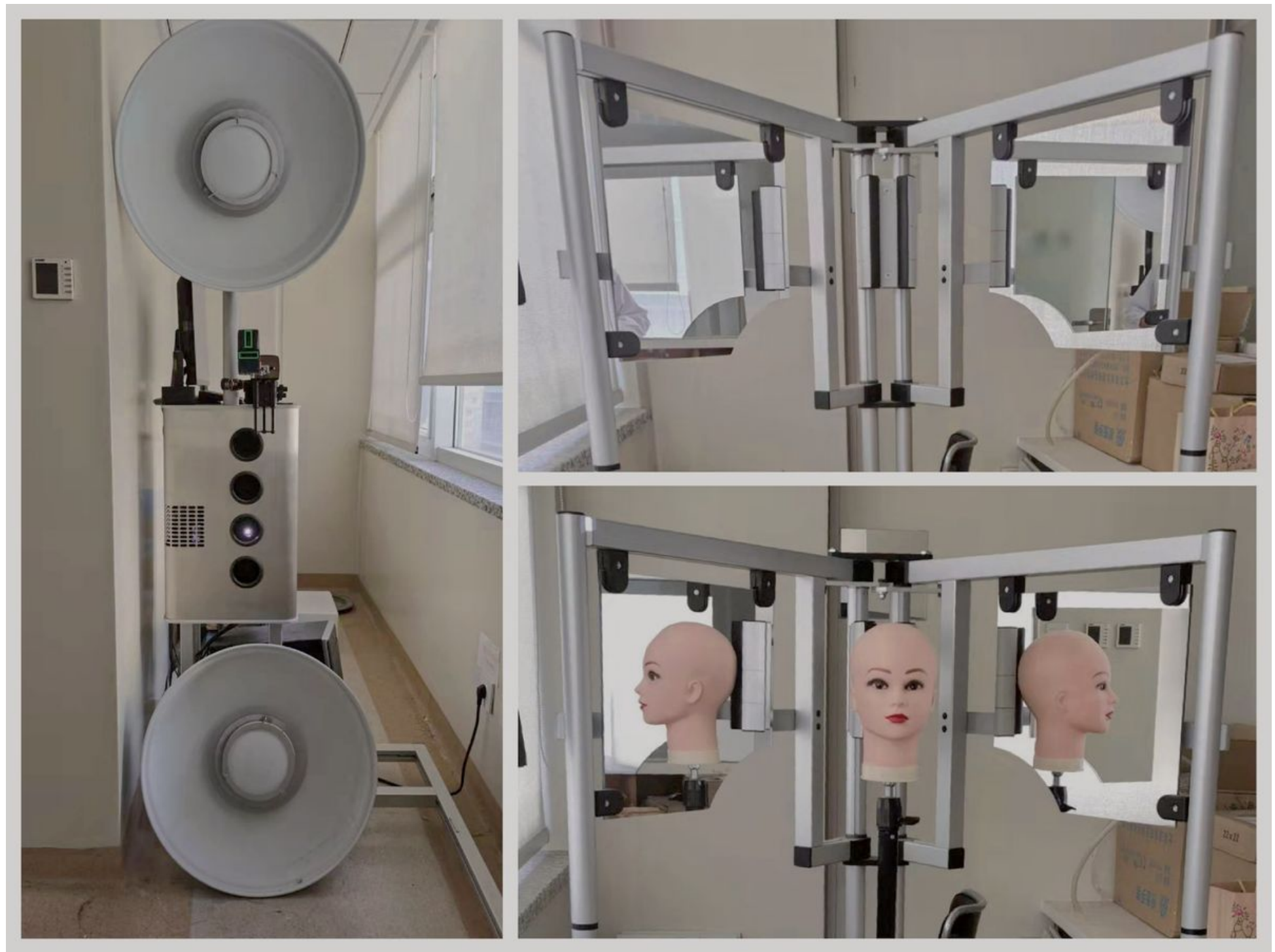

\section{Figure 1}

Three-dimensional (3D) facial scans device.

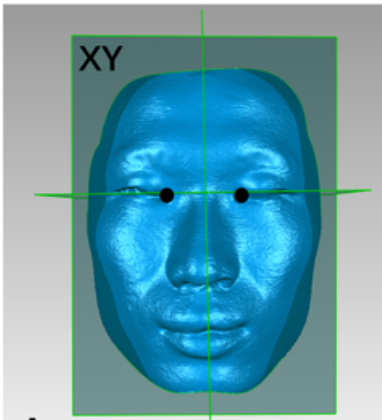

A

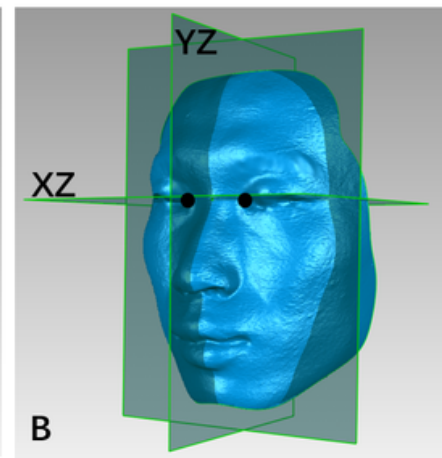

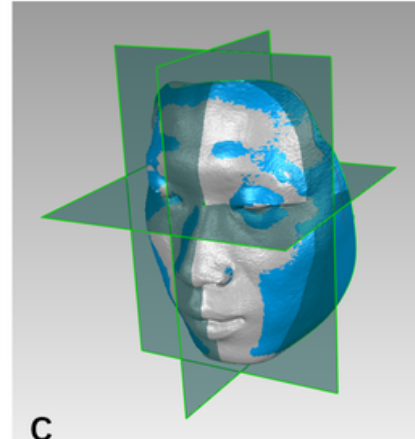

C

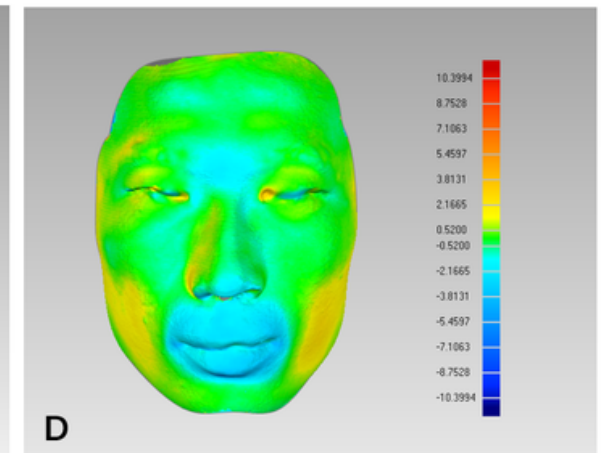

\section{Figure 2}

Construction of the coordinate system (A, B). Superimposition of the two models before (grey) and after (blue) orthodontic treatment with a unified coordinate system (C). Spectrum and color map (blue: inward 
displacement; green: no change; red: outward displacement) showing the three-dimensional superimposition for the two models (D).

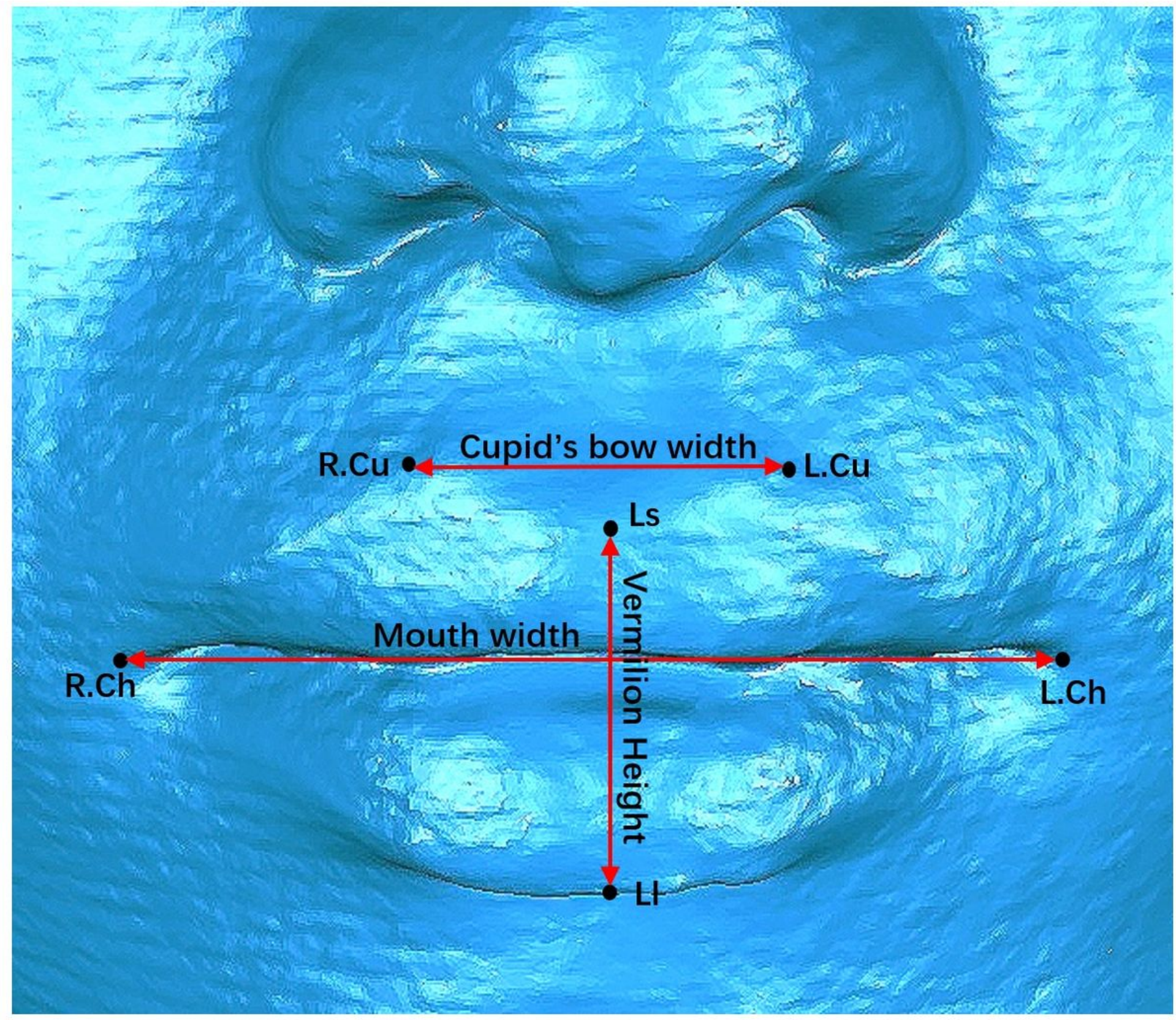

\section{Figure 3}

Six facial landmarks (Ls, LI, R.Cu, L.Cu, R.Ch, L.Ch) (A) and three straight-line distances (vermilion height, cupid's bow width, mouth width). Total vermilion area along the three-dimensional (3D) surface (B). Upper vermilion area along the 3D surface (C). Lower vermilion area along the 3D surface (D). 

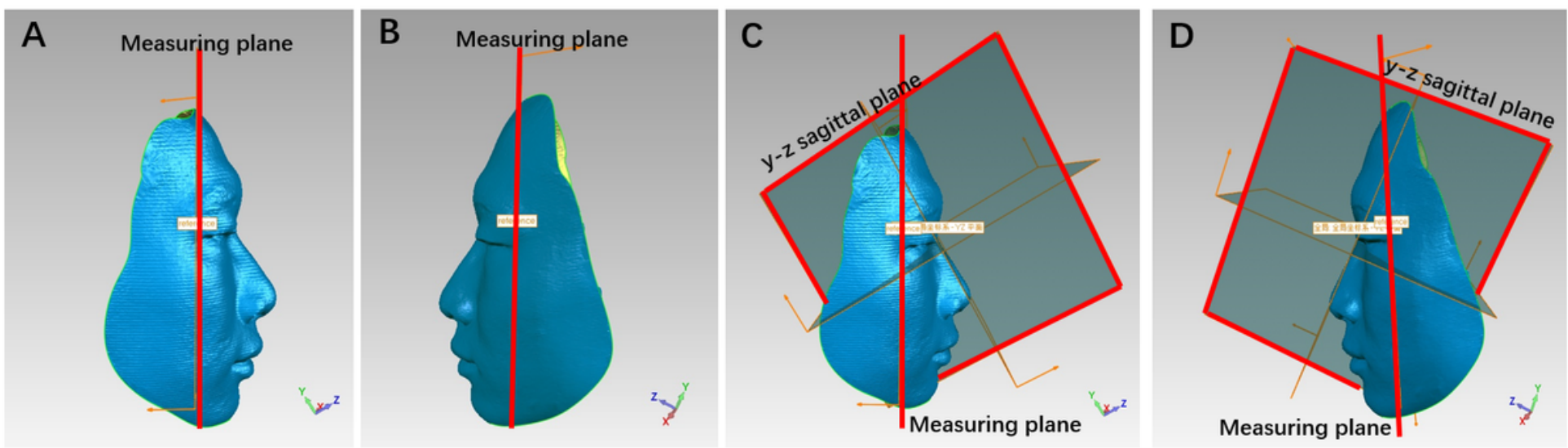

\section{Figure 4}

Illustration of the measuring plane (A, B). Illustration of the measuring plane perpendicular to the $\mathrm{YZ}$ sagittal plane and passing through the bilateral inner canthi point $(C, D)$.

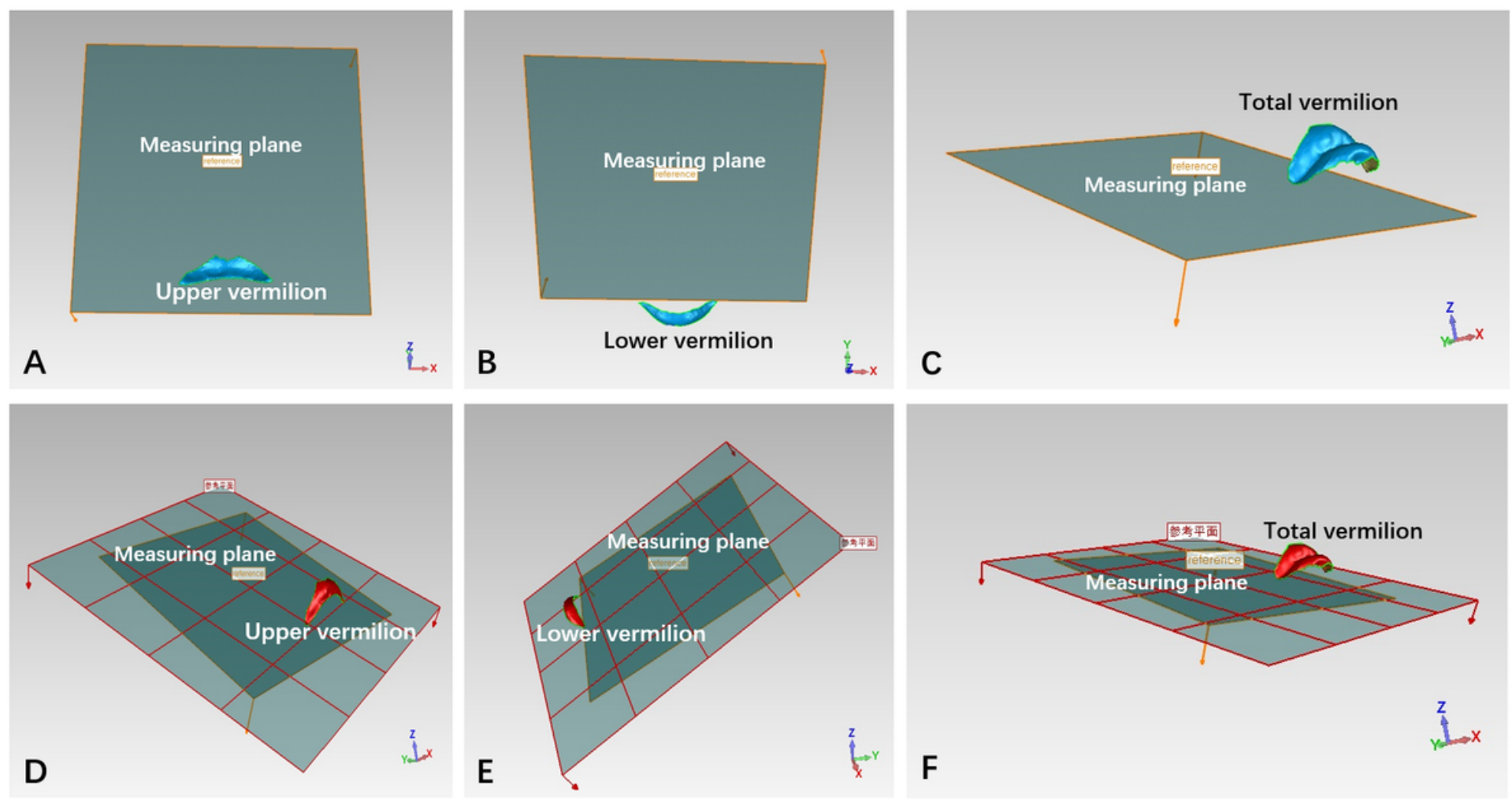

\section{Figure 5}

Illustration of the upper, lower, and total vermilion (blue) marginated manually (A-C). Illustration of the upper, lower, and total vermilion volumes projected to the measuring planes (red) for quantitative analysis $(\mathrm{D}-\mathrm{F})$. 

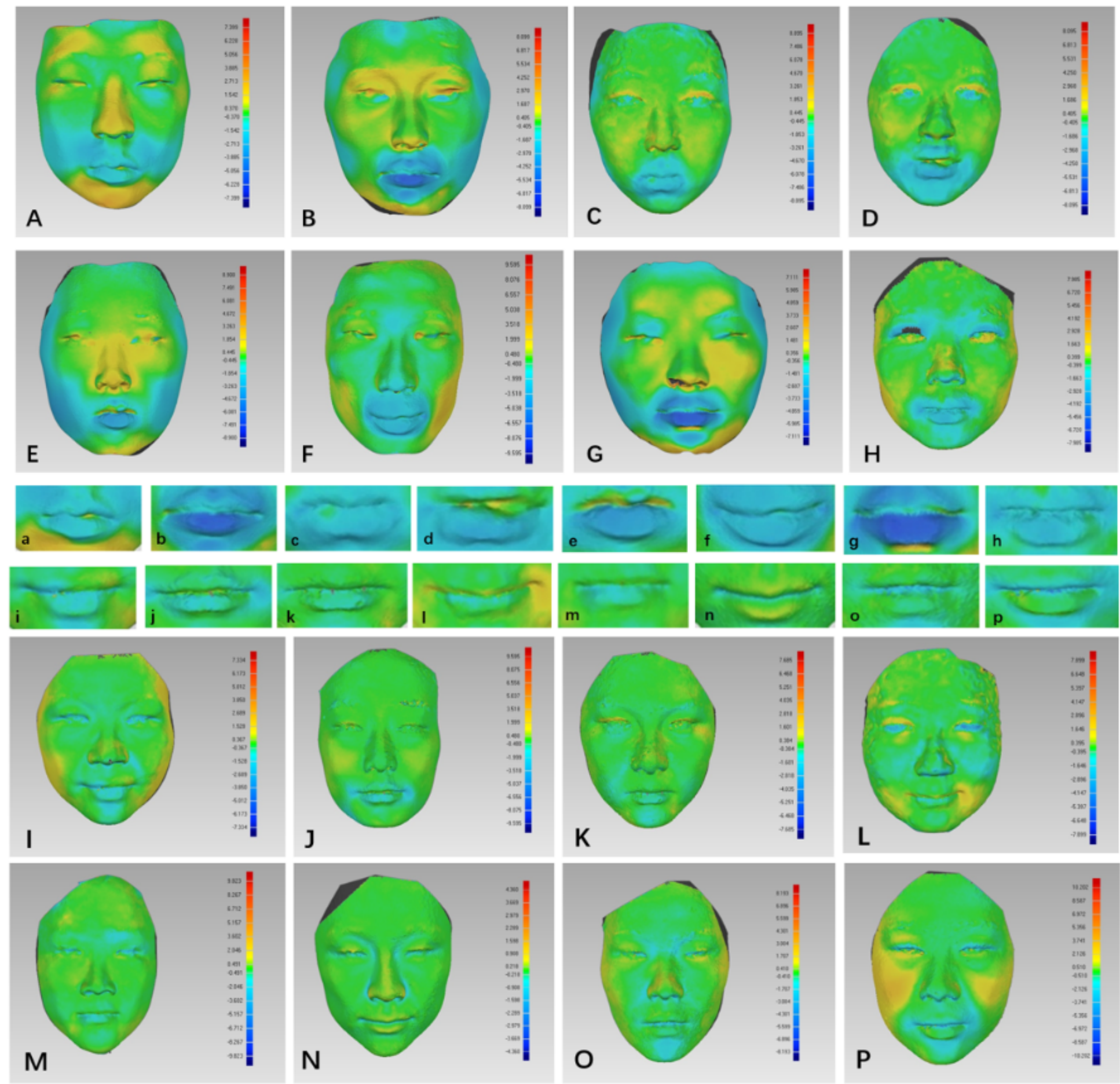

$\mathbf{L}$

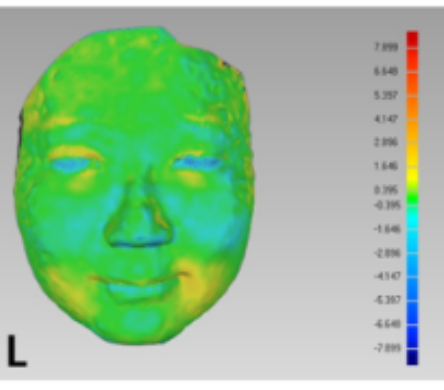

\section{Figure 6}

Superimposed color maps and spectra showing individual variability in facial soft tissues among eight subjects in the treatment group $(A-H)$ and lip vermilions $(a-h)$. Superimposed color maps and spectra showing individual variability in facial soft tissue among eight subjects in the non-treatment group (I-P) and lip vermilions $(i-p)$. Blue: inward displacement; green: no change; red: outward displacement.

\section{Supplementary Files}


This is a list of supplementary files associated with this preprint. Click to download.

- table2.PNG 